\title{
Polyphenols in countercurrent chromatography. An example of large scale separation ${ }^{1}$
}

\author{
A. Berthod*, B. Billardello and S. Geoffroy \\ Laboratoire des Sciences Analytiques, Université de Lyon 1, CNRS UMR 5619, \\ 69622 Villeurbanne Cedex, France
}

\begin{abstract}
Polyphenols are sometimes difficult to separate in classical liquid chromatography. Countercurrent chromatography uses a biphasic liquid system to separate the components of a mixture. A centrifugal field allows to use a liquid stationary phase in an open tube. The phase density difference and the centrifugal field are the only parameters allowing the equilibrium between the two liquid phases. The big advantage of the technique in preparative separation is the dual-mode capability of CCC. The role of the phases can be switched during a run. The mobile phase becomes stationary and vice versa. Then no injected material can be left in the machine. The large scale separations of a flavonoid mixture and two tannin samples are described: choice of the biphasic liquid system, analytical study, scaling-up, injection protocol, two step separation in case of dual-mode use. It was possible to inject $26 \mathrm{~g}$ of a tannin sample in one run.
\end{abstract}

Keywords. Countercurrent chromatography - polyphenol - preparative chromatography - flavonoid tannin.

\section{Introduction}

Polar compounds are commonly found in vegetal extracts. Saponins, cardenolides, iridoids, alkaloids, antibiotics, tannins, flavonoids, quercetrins xanthones, lignans and other polyphenols or sugar or glycoside derivatives are examples of polar compounds very commonly found in vegetal extracts and used as active principles for their pharmacological properties [1]. They are often difficult to separate by HPLC and countercurrent chromatography can be a very useful tool to purify them [2-3].

Countercurrent chromatography (CCC) is the separation technique that uses a liquid stationary phase. The mobile phase is also a liquid phase, i.e., a biphasic liquid system is used. It is important to realize that in most CCC separations there are absolutely no countercurrent flowing liquids. The name of the technique was coined by Yoishiro Ito, its inventor [4]. Publishing more than 250 articles using it, he made the CCC acronym so related to the liquid-liquid centrifugal partition chromatography technique that it is now accepted worldwide [5].

The advantages of the use of a liquid stationary phase are:

- a simple retention mechanism (liquid-liquid partition);

- no irreversible solute adsorption;

- high loading capability;

- large range of polarity;

- little solute modification, degradation or denaturation;

- original selectivity;

- few pH limitations;

- low cost.
The main problem in CCC is to retain the liquid stationary phase when the liquid mobile phase is pushed through it. Centrifugal forces are used in all modern CCC apparatuses $[2,3,5,6]$. The liquid stationary phase is retained in channels by a constant centrifugal field in hydrostatic (HS) apparatuses [7]. It is retained in coils of Teflon tubes by a variable field in hydrodynamic (HD) apparatuses [6]. Another problem is the efficiency reduced to a maximum of one plate per channel for HS apparatuses and two to three plates per coil turn for HD machines. The efficiency of CCC machines ranges from 100 plates to 2000 plates depending on the geometrical characteristic of the machine but also on the biphasic liquid system used and on the operating conditions.

In this work, the separation of polyphenols of vegetal origin is described and the scaling-up of a tannin separation is presented.

\section{Experimental}

\section{Apparatuses}

Three different HD CCC apparatuses were used. They are described in table I. The analytical machine was constructed by the late SFCC company (Société Française de Chromato Colonne) (Éragny, France) that discontinued its CCC machine production in 1992. The two Kromaton, models 2 and 3, preparative machines were built by SEAB (64, rue Pasteur, 94807 Villejuif, France).

1. This work was presented at the chromatographic symposium SEP'99 in Lyon (France), March 31-April 2, 1999. 


\section{Original articles}

Table I. Instrumental characteristics of the hydrodynamic CCC machines.

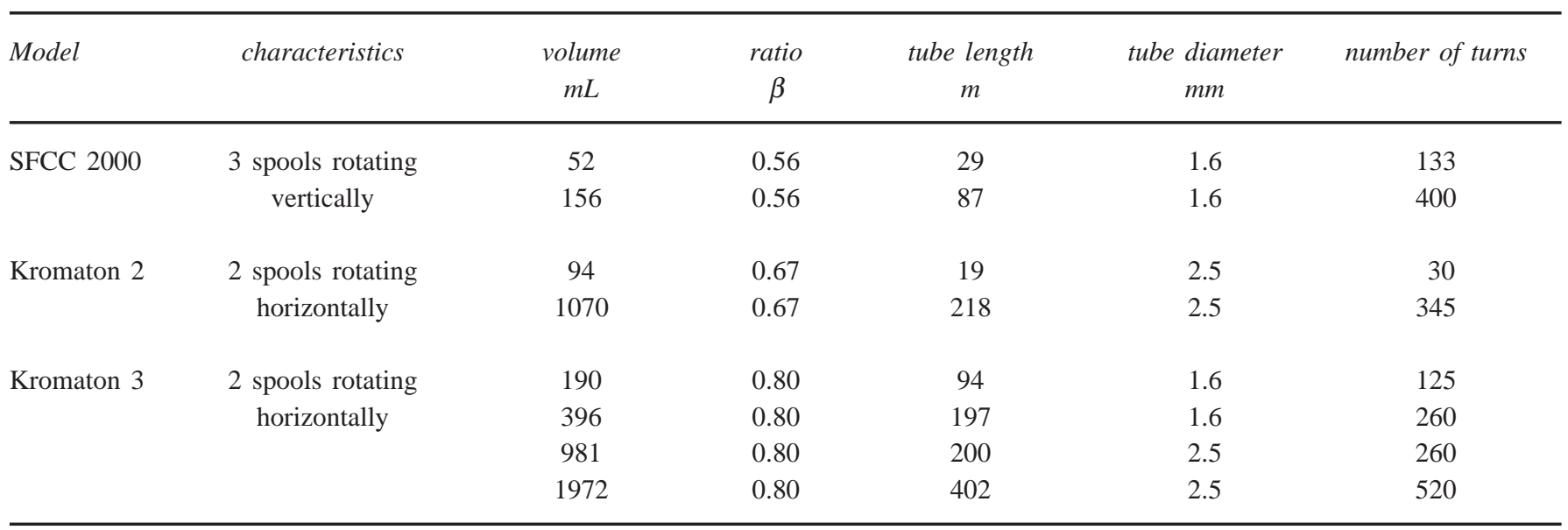

The SFCC machine can work with one or three spools. The spools of the Kromaton machines are coiled with different tubes. The $\beta$ ratio is $r / R$, the ratio of the spool diameter over the rotation diameter (distance between the rotor axis and the spool axis). All three machines have a temperature regulation system.

\section{Other hardware}

Only the column is special in CCC. The peripherical materials, pumps, valves, detectors and recorders or integrators are classical LC hardware. A Shimadzu LC-10AS pump was used for flow rates up to $9.9 \mathrm{~mL} / \mathrm{min}$. A Shimadzu LC8A prep-pump was used when higher flow rates were desired. A Shimadzu SPD-6A UV detector and a Cunow DDL 21 evaporative light scattering detector were used connected to a Shimadzu C-R5A and a C-R6A integrator, respectively. Touzard et Matignon (Dukert, Courtabœuf, France) was the supplier. Thin layer chromatography was done with Merck $10 \times 10$ plates code $60-$ F254.

\section{Solvents}

Ethyl acetate, butanol and butanone (methyl ethyl ketone or MEK), methyl isobutyl ketone (MIBK) were from SDS (Peypin, France). Methanol and acetic acid were obtained from Fluka (Sigma-Aldrich, St Quentin Fallavier, France). They were used as received. The CCC relevant physico chemical properties are listed in table II. Water was deionized and distilled.

\section{Samples}

The Quercetrin extract was obtained from L. Light \& Co. Ltd., Colinbrode, England. The Hamamelis extract was furnished by C.A. Erdelmeier of Willmar Schwabe GmbH, Karlsruhe, Germany. The tannin mixture was the product number 48812, Tannic acid, of Fluka.

\section{Protocol}

Since a centrifugal field is used to hold the liquid stationary phase in the CCC machine, a CCC "column" exists as long as the machine rotor is rotating. Unless otherwise indicated, we used the following protocol to prepare a CCC "column": first the CCC machine is filled at a high flow rate with the liquid phase chosen to be the stationary phase. $200 \mathrm{~min}$ (more than 3 hours) were needed to fill the $2 \mathrm{~L}$ machine at $10 \mathrm{~mL} / \mathrm{min}$. Then the rotor is started at the desired speed and the mobile phase is pushed in the machine in the correct way: from head to tail (top to bottom) if the mobile phase is the denser liquid or from tail to head if it is the lighter phase $[3,6,8]$. The mobile phase equilibrates with the stationary phase turns after turns displacing part of the later. Only the stationary phase is seen exiting the machine. It is collected in a graduated cylinder. When the mobile phase exits out of the machine, the CCC column is ready. The collected stationary phase volume corresponds to $V_{M}$, the volume of the mobile phase inside the machine that is the dead volume. The stationary phase volume retained by the machine is $\mathrm{V}_{\mathrm{S}}=\mathrm{V}_{\mathrm{T}}-\mathrm{V}_{\mathrm{M}}$. These volumes are used to calculate $\mathrm{P}$, the liquid-liquid partition coefficient of an injected solute using its retention volume, $\mathrm{V}_{\mathrm{R}}$ :

$$
\mathrm{V}_{\mathrm{R}}=\mathrm{V}_{\mathrm{M}}+\mathrm{PV}_{\mathrm{S}}=\mathrm{V}_{\mathrm{T}}+(\mathrm{P}-1) \mathrm{V}_{\mathrm{S}}
$$

If the partition coefficient is known, then Eq. (1) allows to predict accurately the retention volume of the solute using the phase volumes inside the CCC "column." Often the retention ability of a CCC machine is rated by the phase retention factor, Sf:

$$
\mathrm{Sf}=\mathrm{V}_{\mathrm{S}} / \mathrm{V}_{\mathrm{T}}
$$

The Sf factor depends on the CCC machine and also on the biphasic liquid system used and the operating conditions [2, 6]. 


\section{Original articles}

Table II. Physico-chemical properties of the solvents used.

\begin{tabular}{|c|c|c|c|c|c|c|c|}
\hline \multirow[t]{2}{*}{ Solvent } & \multirow[t]{2}{*}{$\begin{array}{c}\text { density } \\
\mathrm{g} / \mathrm{cm}^{3}\end{array}$} & \multirow[t]{2}{*}{$\begin{array}{c}\text { viscosity } \\
c P\end{array}$} & \multicolumn{2}{|c|}{$\begin{array}{c}\text { Solubility } \\
\% w / w 20{ }^{\circ} \mathrm{C}\end{array}$} & \multicolumn{3}{|c|}{ Polarity } \\
\hline & & & solvent in water & water in solvent & $\delta$ & Snyder & Reichardt \\
\hline 1-butanol & 0.810 & 2.95 & 7.8 & 20.1 & 27.2 & 3.9 & 60.2 \\
\hline butanone & 0.805 & 0.43 & 24 & 10 & 19.2 & 4.7 & 32.7 \\
\hline ethyl acetate & 0.901 & 0.45 & 8.7 & 3.3 & 18.2 & 4.4 & 22.8 \\
\hline methanol & 0.791 & 0.55 & 4 & 4 & 29.3 & 5.1 & 76.2 \\
\hline water & 0.998 & 1.0 & 4 & 4 & 48.6 & 10.2 & 100 \\
\hline
\end{tabular}

$\delta$ is the Hildebrand parameter. Butanone is also called methyl ethyl ketone (MEK).

\section{Choice of the liquid system}

\section{How to define solvent polarity}

Solvent polarity is roughly related to the interactions between the solvent molecules. If there are no interaction or only Van der Waals interactions between the molecules of a pure solvent, this solvent is considered as apolar, e.g., alkanes, silicon oils, perfluorinated solvents. If there are interactions between the solvent molecules, then the solvent is said to be polar. Different polarity degrees are possible. Weak interactions (dipoles-induced dipoles) will correspond to weakly polar. Strong interactions are found in very polar solvents (hydrogen bonds, ionic interactions in room temperature ionic liquids). At the moment, there are different polarity scales such as the Hildebrand scale based on the solubility parameter, $\delta$, defined as the work necessary to separate two solvent molecules. In chromatography, the Snyder polarity scale, based on the eluting power of the solvent in thin layer chromatography (TLC), is commonly used. In organic synthesis, the Reichardt scale is most often used. It is related on the transition energy for the solvatochromic absorption band of a pyridinium -N-phenoxyde betaine dye. Table II lists the polarity value in the three scales for the solvent used. In the Hildebrand and Reichardt scales, the order is the same: water is the most polar solvent, then methanol, butanol, butanone and ethyl acetate is the less polar. In the Snyder scale, butanol is considered as the less polar solvent. Its high viscosity reduces its eluting power in TLC. Such differences between the different polarity scales are very common [9].

\section{Two or three solvent systems?}

The two solvent biphasic liquid system is the most simple and convenient to use in CCC. It is simple because the mixing of the two solvents will produce the two liquid phases that are one solvent saturated by the other. The butanonewater system forms two liquid phases particularly useful in CCC because of their high polarity. The aqueous denser phase contains $76 \%$ w/w water and $24 \%$ w/w butanone $(28 \% \mathrm{v} / \mathrm{v})$. The upper organic phase contains $10 \% \mathrm{w} / \mathrm{w}$ water and $90 \%$ w/w butanone (92\% v/v). In CCC, the phase that is used as the mobile phase is depleted more rapidly than the other phase. There is no problem to prepare more mobile phase. The mutual saturation of the butanonewater system is fast.

Often it is not possible to find a biphasic system able to perform the desired separation with only two solvents, then three solvents are used: a good solvent for the sample, a second solvent as good for the sample as possible and making a second liquid phase with the first solvent and a third solvent that partitions between the two phases. Ternary phase diagrams are used [7, 10]. The third solvent allows a fine tuning of the partition of the sample components between the two phases. Given the number of possible combinations of three solvents (and more), it can be difficult to find the best biphasic liquid system. General methods to find rapidly the liquid system to perform a good CCC separation were exposed in the literature [11-13]. Figure 1 shows the ternary mass diagram of the water (good solvent)-ethyl acetate (second phase solvent)-butanol system that was used for the separation of tannin samples. Point A locates the composition of the prepared mixture. Points B and C locate the composition of the upper organic and lower aqueous phase obtained when the A mixture is well equilibrated. The BC line is the tie-line corresponding to the A biphasic mixture. Table III lists the corresponding w/w and v/v compositions.

\section{Separation of a flavonoid sample}

The sample called "Quercitrin extract" was fractionated using the simple butanone-water biphasic system. A rapid analysis using the small volume coil of the Kromaton 2 machine showed in $20 \mathrm{~min}$ at $2 \mathrm{~mL} / \mathrm{min}$ (organic mobile phase) that the fractionation of the sample was possible. However, the small coil did not have a sufficient efficiency to resolve fully the sample: only two peaks were obtained.

The large volume coil of the Kromaton 2 machine was then filled with the butanone saturated aqueous stationary phase in more than two hours at $8 \mathrm{~mL} / \mathrm{min}$. Next the rotor was started at 400 RPM and the organic mobile phase was 
Table III. Composition of the A, B and C points of Figure 1.

\begin{tabular}{|c|c|c|c|c|c|c|c|}
\hline \multirow{2}{*}{$\begin{array}{l}\text { Figure } 1 \\
\text { composition }\end{array}$} & \multicolumn{2}{|c|}{ butanol } & \multicolumn{2}{|c|}{ ethyl acetate } & \multicolumn{2}{|c|}{ water ${ }^{*}$} & \multirow{2}{*}{$\begin{array}{l}\text { density } \\
\mathrm{g} / \mathrm{cm}^{3}\end{array}$} \\
\hline & $\% w / w$ & $\% v / v$ & $\% w / w$ & $\% v / v$ & $\% w / w$ & $\% v / v$ & \\
\hline A global mixture & 3.0 & 3.5 & 44.3 & 46.5 & 52.7 & 50 & $\mathrm{~B}$ and $\mathrm{C}$ phases \\
\hline B upper phase & 4.9 & 5.5 & 90.6 & 90.5 & 4.5 & 4 & 0.90 \\
\hline C lower phase & 1.2 & 1.5 & 8.5 & 9.3 & 90.3 & 89.2 & 0.99 \\
\hline
\end{tabular}

* in most experiments, water was actually a $1 \%$ acetic acid solution $(\mathrm{pH} 2.8)$

Table IV. Chromatographic parameters corresponding to Figure 2.

\begin{tabular}{lccccc}
\hline compound & tr & $V r$ & $N$ & $k$ & $P_{\text {butanone/water }}$ \\
& $\min$ & $m L$ & $P_{\text {water/butanone }}$ \\
\hline kaempferol & 67 & 201 & 800 & 0.95 & 10 \\
quercetin & 86 & 258 & 700 & 1.52 & 0.10 \\
kaempferol rhamnoglucoside & 100 & 300 & 640 & 1.90 & 0.16 \\
quercitrin & 129 & 387 & $(600)^{*}$ & 2.75 & 3.9 \\
\hline
\end{tabular}

* estimated value (peak off scale)

$k$ : retention factor calculated as $\mathrm{P}_{\text {water/butanone }} * \mathrm{~V}_{\mathrm{S}} / \mathrm{V}_{\mathrm{M}}$

Experimental CCC conditions described in Figure 2 caption

pumped into the rotating machine at $3 \mathrm{~mL} / \mathrm{min}$ in the tailto-head direction. The stationary phase retention factor was $90.4 \%\left(\mathrm{~V}_{\mathrm{M}}=102 \mathrm{~mL}, \mathrm{~V}_{\mathrm{S}}=968 \mathrm{~mL}, \mathrm{~V}_{\mathrm{T}}=1070 \mathrm{~mL}\right)$. The flavonoid sample was separated in four components with

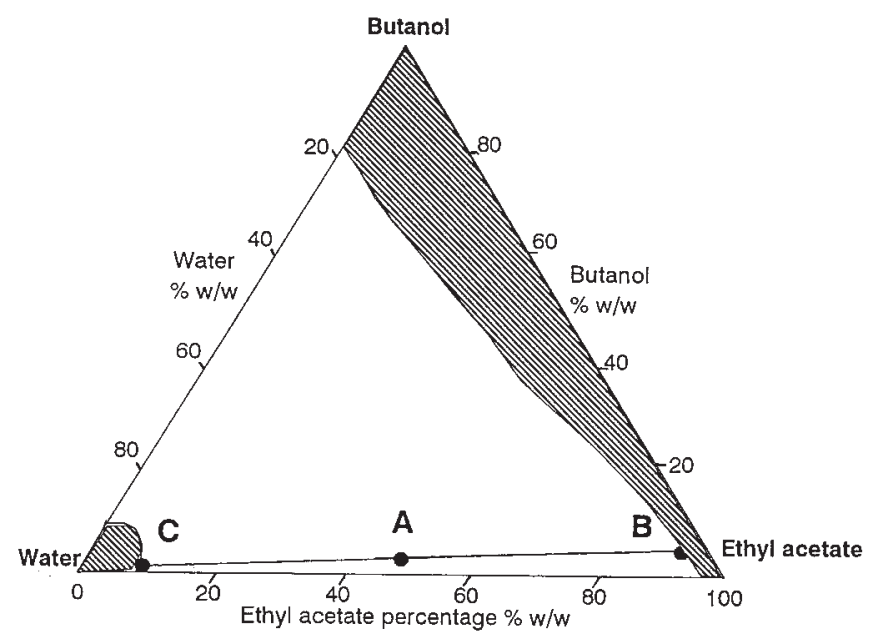

Fig. 1. The ternary mass diagram of the system water-ethyl acetatebutanol at $20{ }^{\circ} \mathrm{C}$. Hatched areas: monophasic compositions (not usable in CCC). Composition A was prepared to perform the tannin separation. It gives Compositions $\mathrm{B}$ and $\mathrm{C}$ whose formulas are listed in Table III.
$650 \mathrm{~mL}$ and in 4 hours. Figure 2 shows the CCC chromatogram of a $0.33 \mathrm{~g}$ injection. The compounds were identified using mass spectrometry as described in a recent article [14].

Table IV lists the chromatographic parameters that can be established from the CCC chromatogram. The retention volumes give the butanone/water partition coefficients of the compounds. These physico chemical parameters are precious data in hydrophobic studies, in quantitative structureactivity relationships (QSAR) and quantitative structureretention relationships (QSRR) in pharmacological or chromatographic studies, respectively. CCC is a very powerful tool to determine accurately the liquid-liquid partition coefficients of solutes $[8,9,15]$.

Table IV and Figure 2 also show that CCC compensates easily its low efficiency by a high selectivity power. Kaempferol and quercetin as well as the rhamnoglucoside derivative and quercitrin differ by only an oxygen atom in the R1 position (Fig. 2). This oxygen atom produces a $60 \%$ and $45 \%$ increase of the $\mathrm{P}_{\text {water/butanone }}$ coefficient, respectively (Table IV). Since the $k$ retention factor is directly proportional to the $\mathrm{P}$ coefficient, the same change is observed on the values. This produced a selectivity factor, $\alpha$, of 1.6 between kaempferol and quercetin and 1.5 between the glucoside derivatives $\left(\alpha=k_{2} / k_{1}\right)$. Such selectivity factors are high enough to produce an almost baseline resolution of the compound with an efficiency of only about 600 plates. 


\section{Original articles}

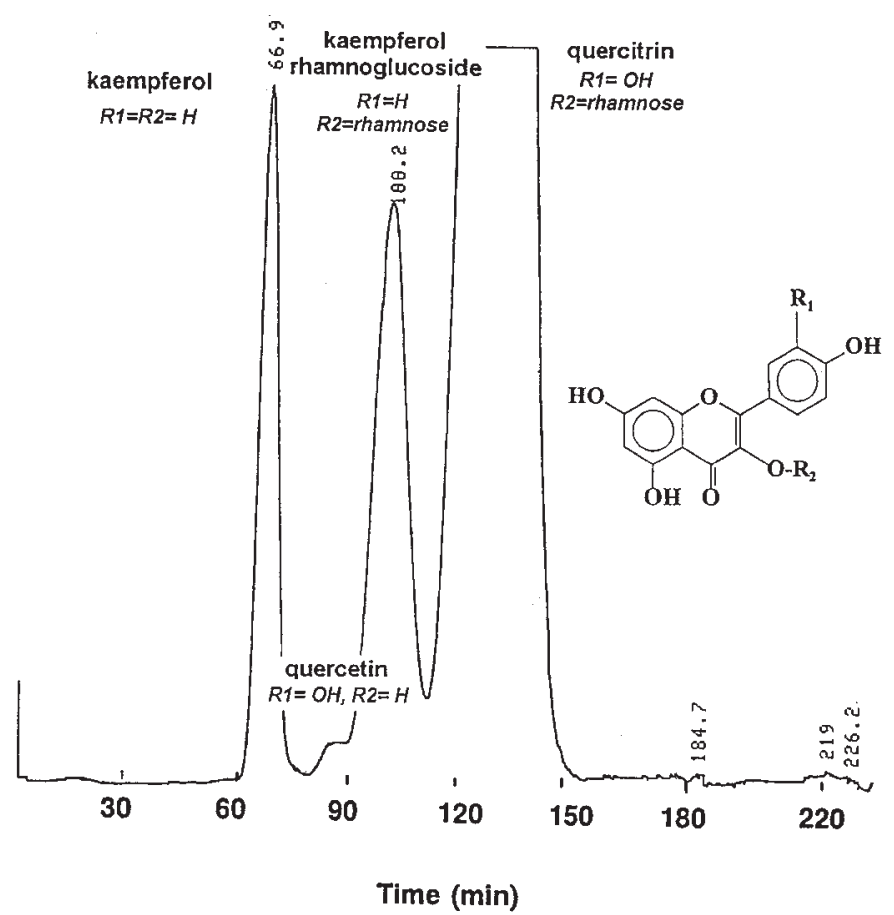

Fig. 2. CCC chromatogram of the quercetin sample. Mobile phase: butanone $3 \mathrm{~mL} / \mathrm{min}$, tail to head. Stationary phase: aqueous. $\mathrm{V}_{\mathrm{T}}=1070 \mathrm{~mL}, \mathrm{~V}_{\mathrm{M}}=102 \mathrm{~mL}, \mathrm{~V}_{\mathrm{S}}=968 \mathrm{~mL}, 400 \mathrm{rpm}$. Injection: volume $9 \mathrm{~mL}$ of a $37 \mathrm{~g} / \mathrm{L}$ solution, mass $330 \mathrm{mg}$. Detection: UV $330 \mathrm{~nm}$.

\section{Mass separation of tannins}

Tannins are polar polyphenols of vegetal origins. They are difficult to separate because in a vegetal extract numerous isomers with a very similar molecular basis coexist $[19,20]$. The use of a liquid-liquid system to fractionate tannin samples produces original selectivities and preparative capabilities that were already used $[3,6-7,16,19,20]$. We present here the separation of the tannin sample sold by Fluka with the name "Tannic acid" product 48812 valid only for the batch \#365176/1 (Feb. 12, 1997). We bought a second $250 \mathrm{~g}$ pot (batch \#395301/1 (May 5, 1998)) that gave different chromatograms. The commercial tannin mixture was used to select the suitable liquid system and investigate the loading capabilities of our hydrodynamic CCC machines.

\section{Optimization using the low volume machine}

The SFCC 2000 machine was used to search for the optimal liquid system. Using and adapting the Margraff approach [12], it was found that the composition A that separates in organic phase B and aqueous phase C (Fig. 1) of the water-butanol-ethyl acetate system was able to partition correctly the tannin sample. Table III lists the exact compositions of the phases.

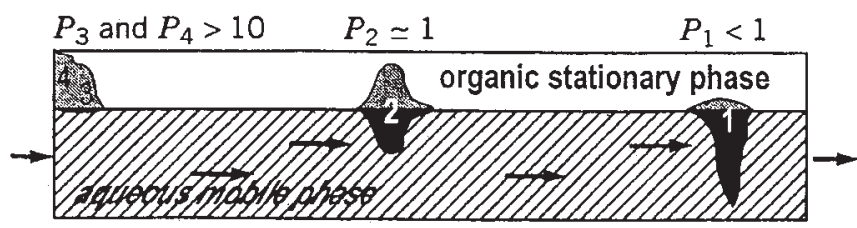

Step 1: the polar solutes are eluted
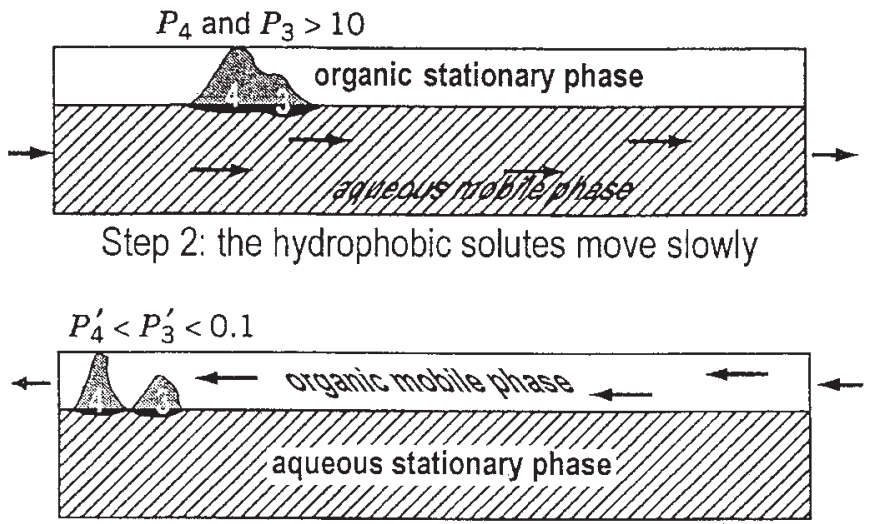

Step 3: the hydrophobic solutes are eluted by the organic phase

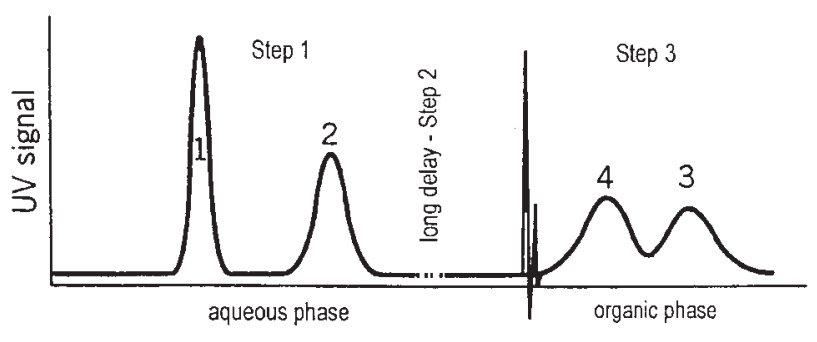

Elution volume, $\mathrm{mL}$

Fig. 3. The dual-mode use of a CCC machine. Steps 1 and 2 are done with the aqueous phase in the head to tail direction. Step 3 is done with the organic light phase in the tail to head direction. Bottom: the corresponding chromatogram.

It was not possible to separate the sample directly. The dual-mode was used. Figure 3 explains graphically this unique way to operate a CCC machine using the liquid nature of the stationary phase [7, 17]. Step 1 is the normal way to use a chromatograph with a mobile, say aqueous, and a stationary, say organic, phase. The aqueous denser phase moves in the head to tail way. In Step 2, it is shown that solutes 3 and 4 would need a huge mobile phase volume to elute. They are hydrophobic with a high affinity for the organic apolar phase. The answer to elute them is to switch the phase role: the stationary phase becomes the mobile phase and vice versa. Step 3 shows that solutes 3 and 4 are easily eluted by the organic mobile phase in the opposite tail to head way. Furthermore, the separation initiated in Step 2 is improved in Step 3 since 4 moves faster than 3 with the organic mobile phase. The advantages of the dual mode use 
were evidenced in the partition coefficient determination $[8$, $15]$ and general uses of CCC [7, 18].

The tannin sample was fractionated in 12 peaks. 7 peaks eluted in the head to tail way with an aqueous mobile phase of composition $\mathrm{C}$ (Fig. 1). 5 more peaks eluted in the reversed tail to head way with the composition $\mathrm{B}$ organic mobile phase (not shown by Figure 4, see Table V). The paramount advantage of the dual mode use of CCC is that nothing can be left in the machine if the organic phase volume, $\mathrm{V}_{\text {org }}$, pushed in the reversed tail to head way is higher than:

$$
\mathrm{V}_{\text {org }} \geq \mathrm{V}_{\mathrm{aq}} \mathrm{V}_{\mathrm{S}} /\left(\mathrm{V}_{\mathrm{aq}}-\mathrm{V}_{\mathrm{M}}\right)
$$

in which the subscript aq, $\mathrm{S}$, and $\mathrm{M}$ refer to the volume of aqueous phase pushed in the head to tail direction after the sample injection in Steps 1 and 2 (Fig. 3), the volume of organic phase that was retained and stationary in Steps 1 and 2 , and the volume of aqueous phase that was mobile in Steps 1 and 2, respectively. The $\mathrm{V}_{\mathrm{S}}$ volume of organic phase in the machine should remain the same during Step 3. Usually it does $[7,9,15,17,18,20]$.

Tannins are very sensitive to small $\mathrm{pH}$ changes, they oxidize readily in basic media. To stabilize the aqueous phase $\mathrm{pH}$ at a 2.8 value, $1 \% \mathrm{v} / \mathrm{v}$ acetic acid was added to water before preparing the biphasic system. Table V lists the retention values and partition coefficients corresponding to the peaks obtained. The partition coefficients listed correspond to the affinity of the compounds for the organic phase $\left(\mathrm{P}_{\text {org/aq }}\right)$. The affinity for the aqueous phase would be measured by the inverse: $\mathrm{P}_{\mathrm{aq} / \mathrm{org}}=1 / \mathrm{P}_{\text {org/aq. }}$. The peak elution order from 1 to 7 (Fig. 4) corresponds to the increasing $\mathrm{P}_{\text {org/aq }}$ order. The $\mathrm{P}$ values are obtained by Eq. 1 . The mode inversion is done after a volume $\mathrm{V}_{\text {aq }}$ of aqueous phase is pumped in the head to tail direction. Then, the mobile phase becomes the organic phase pumped in the tail to head direction (Fig. 3). The peaks elute in decreasing $\mathrm{P}_{\mathrm{aq} / \text { org }}$ order (Table V). The $\mathrm{P}$ value is calculated as $\mathrm{P}=\mathrm{V}_{\mathrm{aq}} / \mathrm{V}_{\mathrm{R}}[15,17]$. The volume of organic phase needed to elute all the hydrophobic tannins out of the CCC machine is $95 \mathrm{~mL}$ (Eq. 3). When $100 \mathrm{~mL}$ of organic phase are pumped in the tail to head direction, it is certain that no injected compound remains in the machine. This experiment shows that CCC was able to separate peaks corresponding to compounds with $\mathrm{P}$ values ranging from 0.08 (hydrophilic) to 67 (less polar). However tannins are so complex that the separated peaks did not correspond to a single component. A thin layer chromatography plate was done for every peak. All of them, except maybe peak \#8 showed more than one spot after elution with an AcOEt-MIBK-Acetic acid (50-50-1 v-v-v) mobile phase and revelation with a $\mathrm{FeCl}_{3}$ pulverization (blue spots). Evaporating the collected fraction, it was found that Peak \#8 contained more than $80 \%$ of the injected mass.

\section{Scaling-up the separation}

The tannin separation was scaled-up injecting larger amounts on the same machine. A $30 \mathrm{~g} / \mathrm{L}$ solution was
Table V. Chromatographic parameters of the tannin separation presented in Figure 4.

\begin{tabular}{lcccc}
\hline peak & $\begin{array}{c}t r \\
\min \end{array}$ & $\begin{array}{c}V_{R} \\
m L\end{array}$ & $P$ & calculation \\
& & & \\
\hline 1 & 40.0 & 80.1 & 0.08 & \\
2 & 0.81 & 258 & 0.22 & $P=\left(V_{R}-V_{M}\right) / V_{S}$ \\
3 & 0.805 & 111.3 & 0.48 & $\mathrm{~V}_{\mathrm{M}}=74 \mathrm{~mL}$ \\
4 & 74.4 & 148.7 & 0.96 & $\mathrm{Vs}=78 \mathrm{~mL}$ \\
5 & 89.6 & 179.2 & 1.35 & $\mathrm{~V}_{\mathrm{T}}=152 \mathrm{~mL}$ \\
6 & 0.998 & 289.0 & 2.76 & $\mathrm{Sf}=52 \%$ \\
7 & 193.6 & 387.2 & 4.02 &
\end{tabular}

Mode inversion after 215 min (not shown in Figure 4)

\begin{tabular}{lcccc}
8 & 3.21 & 6.42 & 67.0 & \\
9 & 5.48 & 10.96 & 39.2 & $P=V_{a q} / V_{R}$ \\
10 & 9.31 & 18.62 & 23.1 & $\mathrm{~V}_{\text {aq }}=430 \mathrm{~mL}$ \\
11 & 23.8 & 47.6 & 9.0 & \\
12 & 37.8 & 75.6 & 5.7 & \\
\hline
\end{tabular}

prepared by dissolving the exactly weighted amount of tannins in the adequate aqueous phase volume. A $300 \mathrm{~g} / \mathrm{L}$ solution was prepared by dissolving $30 \mathrm{~g}$ of the tannin sample in a mixture of $5 \mathrm{~mL}$ of aqueous and $5 \mathrm{~mL}$ of organic phase. The resulting solution was monophasic. Figure 4 shows the chromatogram obtained when $30 \mathrm{mg}(1 \mathrm{~mL}$ of the $30 \mathrm{~g} / \mathrm{L}$ solution, Fig. 4-A) and $750 \mathrm{mg}$ ( $2.5 \mathrm{~mL}$ of the $300 \mathrm{~g} / \mathrm{L}$ solution, Fig. 4-B) were injected in the $150 \mathrm{~mL}$ machine. The loading capability of the CCC machine are demonstrated. Almost no broadening can be seen on Peaks \#6 and \#7 between the $30 \mathrm{mg}$ and $750 \mathrm{mg}$ injections. Peaks 1, 4 and 5 broaden somewhat, but they correspond to a twenty-time higher amount of tannins. The retention volumes are not affected by the concentration increase.

The $300 \mathrm{~g} / \mathrm{L}$ solution had an elevated viscosity. Injection loops with volumes higher than $2.5 \mathrm{~mL}$ could be made but a pressure increase due to the sample viscosity limited their use (1/16" tubing, $0.5 \mathrm{~mm}$ i.d.). The Kromaton CCC machine can use $1.6 \mathrm{~mm}$ and $2.5 \mathrm{~mm}$ i.d. tubing in its injection port. A $66 \mathrm{~mL}$ loop was made allowing to inject $20 \mathrm{~g}$ when the $300 \mathrm{~g} / \mathrm{L}$ solution was prepared. The $2 \mathrm{~L}$ spool configuration was equilibrated with the Table III liquid system. The organic phase retention ratio was only $26 \%$ at $420 \mathrm{rpm}$. The $66 \mathrm{~mL}$ injection of the viscous tannin solution produced an important stationary phase leak. The injected plug forms a piston pushing the biphasic liquid system out of the machine and no separation occurs. After some unsuccessful injections, the protocol was modified as follows: $17 \mathrm{~min}$ were needed to inject the sample at $4 \mathrm{~mL} / \mathrm{min}$, then $120 \mathrm{~mL}$ of aqueous mobile phase were further introduced in the machine at $4 \mathrm{~mL} / \mathrm{min}$ for 30 more minutes, next the flow rate was stopped, the mode valve was switched in the opposite way (tail to head) and the flow rate was resumed for 

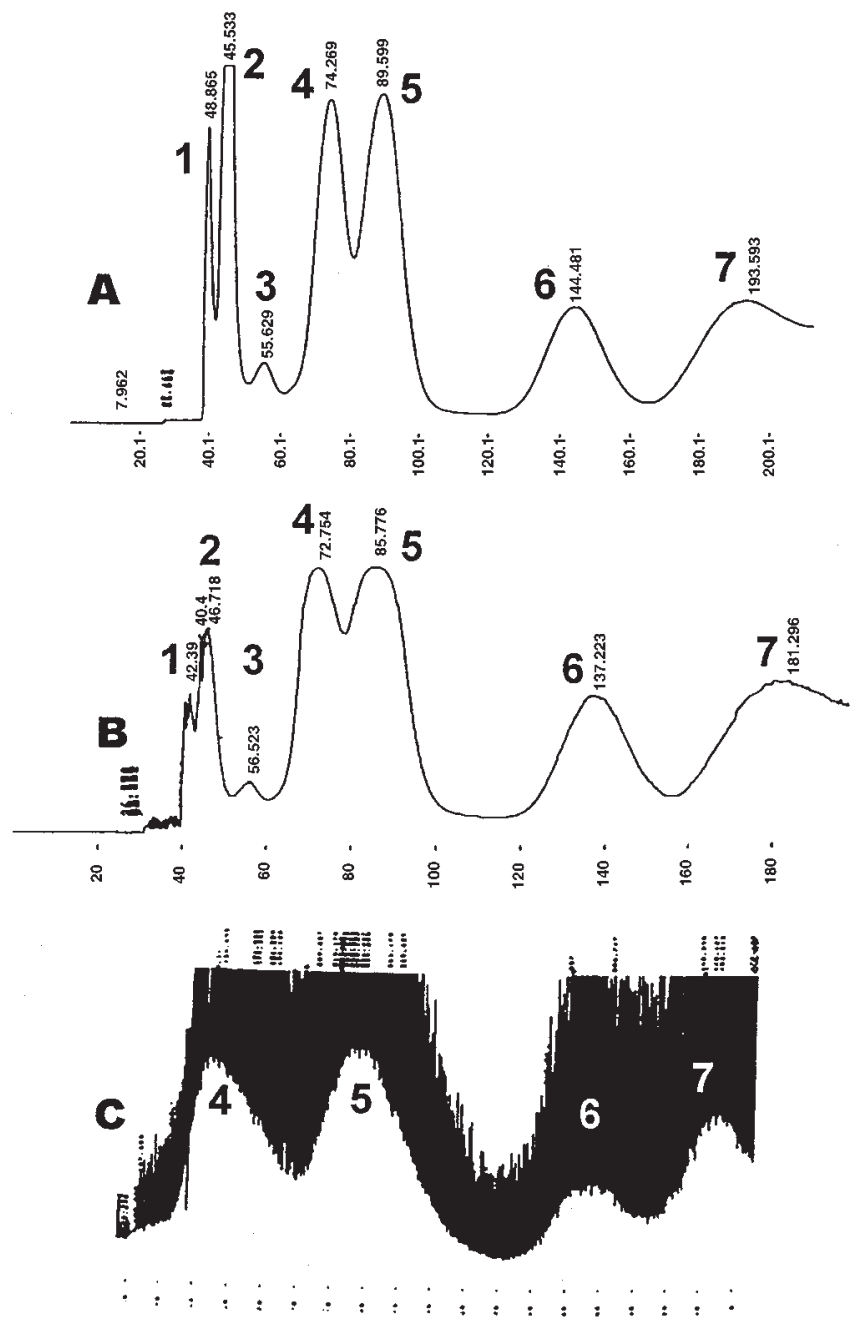

Fig. 4. Separation of a vegetal tannin sample. A- separation of $30 \mathrm{mg}(1 \mathrm{~mL}$ of $30 \mathrm{~g} / \mathrm{L}$ solution) by the SFCC machine $\left(\mathrm{V}_{\mathrm{T}}=152 \mathrm{~mL}\right)$. B- Scaling-up with the same machine. Separation of $0.75 \mathrm{~g}$ injected in $2.5 \mathrm{~mL}$ of a $300 \mathrm{~g} / \mathrm{L}$ solution. $800 \mathrm{rpm}$, aqueous mobile phase in the head to tail direction, $2 \mathrm{~mL} / \mathrm{min}, \mathrm{Sf}=56 \%$. C- Large scale separation (20 g in $66 \mathrm{~mL}$ of a $300 \mathrm{~g} / \mathrm{L}$ solution) with the Kromaton 3 2L-preparative machine. $420 \mathrm{rpm}, 4 \mathrm{~mL} / \mathrm{min}$, $\mathrm{Sf}=26 \% .280 \mathrm{~nm}$ UV detection. Liquid system: see Table III.

$10 \mathrm{~min}$. This was designed to dissolve the plug of injected phase and to force the sample to partition between the two liquid phases. After that, the flow rate was stopped again, the machine was let to equilibrate for $5 \mathrm{~min}$, the mode valve was switched back in the correct position (head to tail) and the flow rate was resumed at $4 \mathrm{~mL} / \mathrm{min}$. Figure $4-\mathrm{C}$ shows part of the obtained chromatogram. The retention volume of Peak \#7 was $3.3 \mathrm{~L}$ (almost 14 hours retention time).

The important noise observed on the chromatogram 4-C is due to a significant stationary phase leak. Microdroplets of stationary phase pass through the detector producing the numerous spikes making the noise. The organic stationary phase loss was measured to be $20 \mathrm{~mL} / \mathrm{h}$. The Sf phase retention ratio dropped from an initial $26 \%$ value $\left(\mathrm{V}_{\mathrm{S}}=515 \mathrm{~mL}\right)$ to $\mathrm{Sf}=11 \%\left(\mathrm{~V}_{\mathrm{S}} \sim 210 \mathrm{~mL}\right)$ at the end of the head to tail phase of the preparative tannin separation. The injection protocol and the separation done with a continuously decreasing stationary phase volume modified somewhat the chromatogram aspect. It is still possible to note that the peak looks gaussian with minimal distortion (Fig. 4-C). This was not true during the tail to head phase of the separation. A huge and broad peak was obtained, but it was not possible to locate the 5 peaks obtained with the analytical CCC machine. This is likely due to the stationary phase volume reduction. The stationary phase loss could easily be reduced or even stopped by increasing the centrifugal field (rotation speed). The apparatus we used was a prototype limited to a $450 \mathrm{rpm}$ maximum rotation speed. This rotation speed may be not enough to create a centrifugal field song enough to retain correctly polar biphasic liquid systems. The rotor mass is in the $30 \mathrm{~kg}$ range when loaded with the liquids. The overall rotation diameter (rotor and spool) is in the half meter range. It is obviously difficult to make a well-balanced rotor of this size and mass. The small vibrations generated by the minute out-of-balance may became dramatic and dangerous when the rotation speed is increased. This mechanical problem is under investigation and it should be resolved since a 600 to $800 \mathrm{rpm}$ speed ( 2 to 4 times higher centrifugal field) seems desirable.

The protocol established with the commercial tannin sample was applied to a real sample, a Hamamelis extract, proposed by Schwabe (Germany). This extract had been purified to refine the glucoside hamameli-tannin. It contained a large majority of the desired tannin along with non desired tannins. The analytical separation of the Hamamelis tannin sample with the Table III biphasic liquid system showed 4 peaks: 3 impurities $(25 \% \mathrm{w} / \mathrm{w})$ and a major peak containing about $3 / 4$ of the injected mass. The UV spectra of the collected fractions were too similar to allow the characterization of the hamameli-tannin. We suppose that the major tannin was the desired one. The preparative separation with the 2L Kromaton machine was done in two steps: 11 hours $(2.6 \mathrm{~L})$ in the head to tail direction with the aqueous mobile phase and 6 hours $(1.4 \mathrm{~L})$ in the opposite tail to head direction with the organic mobile phase. The major peak (more than $70 \%$ of the injected mass) was the first to elute with a $390 \mathrm{~min}$ retention time or $1560 \mathrm{~mL}$ retention volume. If this peak was Hamameli-tannin, it was completely eluted in $420 \mathrm{~min}(1.7 \mathrm{~L})$. If the other tannins are not wanted, it is possible to stop the CCC machine after $420 \mathrm{~min}$, to empty it rapidly using a compressed gas and to start a new purification of $25 \mathrm{~g}$. With these conditions, the throughput of the production can be estimated to $20 \mathrm{~g}$ in 8 hours or $2.5 \mathrm{~g} / \mathrm{h}$.

\section{Conclusion}

The preparative capability of CCC is one more time demonstrated. Large volume CCC machines that are appearing on the market, are able to separate large mass of sample in one 


\section{Original articles}

run giving throughput comparable with classical prep-LC. The original selectivity obtained with the biphasic liquid system chosen is associated with the paramount advantage of the guaranteed total recovery of the injected mass. Other chromatographic modes not presented in this work, such as displacement chromatography or $\mathrm{pH}$ zone refining [19], are able to separate even higher mass of sample in one run. As the reliability of the CCC machines increases (and the noise they generate decreases !), they will be more and more used as powerful tools to purify large mass of compounds. CCC machines are already parts of production processes in the pharmaceutical industry.

\section{Acknowledgments}

François De La Poype, Director of the S.E.A.B, company is gratefully acknowledged for the loan of a prototype of the Kromaton 3 CCC apparatus. C.A.J. Erdelmeier of the Schwabe GmbH company (Karlsruhe, Germany) is thanked for the gift of $80 \mathrm{~g}$ of a refined Hamamelis extract.

\section{References}

1. Hostettmann, K.; Hostettmann, M.; Marston, A. Natural Products Reports 1984, 471-481.

2. Conway, W.D. Chapter 1 in Modern Countercurrent Chromatography, ACS Symposium Series 1993, 593, 1-14.

3. Ito, Y.; Conway, W.D., "High Speed Countercurrent Chromatography", Chemical Analysis, J. Wiley, New York, 1996, 132 .

4. Ito, Y.; Bowman, R.L. Science 1970, 167, 281-283.
5. Ito, Y. "CCC" in J. Chromatogr. Library, Heftmann, E. Ed., Elsevier, Amsterdam, 1992, 51A, 69-105.

6. Conway, W.D. Countercurrent Chromatography, Apparatus, Theory and Applications; VCH Publishers: Weinheim, 1989.

7. Foucault, A.P. (Ed.) "Centrifugal Partition Chromatography" Chromatographic Science Series 1995, 68.

8. Berthod, A. Spectra 2000 1989, 144, 34-38 and 1992, 169, 2224.

9. Berthod, A. in Foucault, A.P. (Ed.) "Centrifugal Partition Chromatography" Chromatographic Science Series, Chapter 7, 1995, 68, 167-197.

10. Berthod, A.; Duncan, J.D.; Armstrong, D.W. J. Liq. Chromatogr. 1988, 11, 1171-1185.

11. Foucault, A.P.; Chevolot, L. J. Chromatogr. A 1998, 808, 322.

12. Margraff, R. in Foucault, A.P. (Ed.) "Centrifugal Partition Chromatography" Chromatographic Science Series, Chapter 4, 1995, 68, 80-87.

13. Oka, F.; Oka, H.; Ito,Y. J. Chromatogr. A 1991, 538, 99-111.

14. Berthod, A.; Talabardon, K.; De La Poype, F.; Erdelmeier, C.A.J. J. Liq. Chromatogr. 1998, 21, 3003-3019.

15. Berthod, A.; Carda-Broch, S.; Alvarez-Coque, M.C.G. Anal. Chem. 1999, 71, 879-888.

16. Marston, A.; Hostettman, K. J. Chromatogr. A 1994, 658, 315341.

17. Menges, R.; Bertrand, G.; Armstrong, D.W. J. Liq. Chromatogr. 1990, 13, 3061-3077.

18. Agnely, M.; Thiebaut, D. J. Chromatogr. A 1997, 790, 17-30.

19. Maillard, M.; Marston, A.; Hostettmann, K. in Ito, Y.; Conway, W.D., Chemical Analysis; J. Wiley: New York, 1996, 132, Ch. 7, 179-223.

20. Okuda, T; Yoshida, T.; Hatano, T.; Yazaki, K.; Kira, R.; Ikeda, Y. J. Chromatogr. 1986, 362, 375-389. 